\title{
Flow field measurement around vortex cavitation
}

\author{
P. C. Pennings ${ }^{1} \cdot$ J. Westerweel $^{1}$ - T. J. C. van Terwisga ${ }^{1}$
}

Received: 6 July 2015 / Revised: 15 September 2015 / Accepted: 17 October 2015 / Published online: 7 November 2015

(C) The Author(s) 2015. This article is published with open access at Springerlink.com

\begin{abstract}
Models for the center frequency of cavitatingvortex induced pressure-fluctuations, in a flow around propellers, require knowledge of the vortex strength and vapor cavity size. For this purpose, stereoscopic particle image velocimetry (PIV) measurements were taken downstream of a fixed half-wing model. A high spatial resolution is required and was obtained via correlation averaging. This reduces the interrogation area size by a factor of 2-8, with respect to conventional PIV measurements. Vortex wandering was accounted for by selecting PIV images for a given vortex position, yielding sufficient data to obtain statistically converged and accurate results, both with and without a vapor-filled vortex core. Based on these results, the loworder Proctor model was applied to describe the tip vortex velocity outside the viscous core, and the cavity size as a function of cavitation number. The flow field around the vortex cavity shows, in comparison with a flow field without cavitation, a region of retarded flow. This layer around the cavity interface is similar to the viscous core of a vortex without cavitation.
\end{abstract}

\section{Introduction}

The design of a propeller for high efficiency is often constrained by cavitation. As the effects of sheet cavitation are relatively well understood, counter measures against harmful expressions of sheet cavitation are taken in the design

\footnotetext{
P. C. Pennings

p.c.pennings@tudelft.nl

1 Department of Mechanical, Maritime and Materials Engineering, Delft University of Technology, Mekelweg 2, 2628 CD Delft, The Netherlands
}

stage. These involve the smooth transport of vapor into the tip vortex, leading harmful implosions away from the propeller surface. The tip vortex cavity dynamics are not directly related to the propeller rotation rate. The excitation of this cavity often leads to high amplitude broadband pressure fluctuations between the fourth and seventh blade rate frequency (Wijngaarden et al. 2005).

To better understand the sound production from vortex cavitation, a model for the waves on a tip vortex cavity was developed and was shown to be able to describe the interface dynamics in detail (Pennings et al. 2015). As a result, a condition could be predicted where a cavity resonance frequency could be amplified to produce high amplitude sound, as reported by Maines and Arndt (1997).

The part that remains to be validated is a vortex model for the cavity size and cavity angular velocity. It has been shown that a Lamb-Oseen vortex model poorly estimates the cavity size as a function of cavitation number (Pennings et al. 2015). It is possible that overestimation of the vortex peak azimuthal velocity could have led to an overestimation of the cavity size. The main goal of the present study was to configure a simple low-order vortex model, to serve as an input into a vortex cavity wave dynamics model, to describe the tip vortex resonance frequency. It is based on the following steps.

1. Model the tip vortex velocity field without cavitation (further referred to as wetted vortex)

2. Model the cavity size as a function of cavitation number based on wetted vortex properties

3. Show the difference in velocity field around a wetted and cavitating vortex

4. Obtain the cavity angular velocity value that gives the best correlation to the tip vortex cavity resonance frequency 
To achieve these goals, the velocity field was measured around a tip vortex close to the viscous core, in the presence and absence of cavitation. High-resolution measurements on the wing tip vortices have been performed in numerous other studies. Point-wise laser Doppler velocimetry was used on a stationary wing tip vortex, in a chronological order (Higuchi et al. 1987; Arndt et al. 1991; Arndt and Keller 1992; Fruman et al. 1995; Boulon et al. 1999). Later the same method was used to measure the flow field including the tip vortices trailing a rotating propeller (Felli et al. 2009, 2011; Felli and Falchi 2011).

Planar particle image velocimetry (PIV) was used on a wing at low Reynolds numbers by Zhang et al. (2006) and Lee (2011). At higher Reynolds numbers, real model aircraft wing tip vortex velocities were measured by Scarano et al. (2002). Typical problems encountered in PIV measurements on tip vortices were shown at the First International PIV Challenge (Stanislas et al. 2003).

Stereo PIV (SPIV) was applied at low Reynolds numbers of $10^{4}$ by del Pino et al. (2011). SPIV measurements of Chang et al. (2011) and Dreyer et al. (2014), similar to the present study, both focused on the effect of the velocity field on vortex cavitation, but no velocities were measured in the presence of cavitation.

In the present study using SPIV, the interrogation area size was limited by the particle seeding density, due to the small field of view around the tip vortex. To accurately determine the velocity around the viscous core of a tip vortex, a high spatial resolution and a high accuracy with respect to spatial gradients are necessary. This requires much smaller interrogation areas than the typical $32 \times 32$ pixels.

A method is proposed for the high-resolution timeaveraged velocity measurement around vortex cavitation. First, large interrogation areas were used to identify the vortex center in the instantaneous vector fields. This was necessary due to vortex center wandering. Similar vortex center identification was used by Graftieaux et al. (2001), del Pino et al. (2011), Bhagwat and Ramasamy (2012) and Dreyer et al. (2014). Second, the original PIV images were grouped according to their vortex center position. Third, the images with the same vortex center position were evaluated together, according to the correlation averaging method introduced by Meinhart et al. (2000). This increases the correlation quality based on the number of images used, while allowing a smaller interrogation area size.

Section 2 describes the SPIV setup, and the details on the SPIV analysis, including the high-resolution time-averaging method and the estimation of the cavity diameter, are given in Sect. 3. The four principal parts of the study are evaluated in Sect. 4. Finally, the limitations of the models and the conclusions are presented in Sects. 5 and 6 , respectively.

\section{Experimental setup}

The experiments were performed in the cavitation tunnel at the Delft University of Technology. The tunnel details can be found in Foeth (2008) with the recent modifications described in Zverkhovskyi (2014). All measurements in the present study were performed at $6.8 \mathrm{~m} / \mathrm{s}$ with typical fluctuations of $\pm 0.5 \%$. The cross section of the test section was $0.30 \times 0.30 \mathrm{~m}^{2}$ at the inlet and $0.30 \times 0.32 \mathrm{~m}^{2}$ at the outlet. The vertical direction was extended gradually from inlet to outlet, to compensate for the growth of the boundary layer, in order to facilitate a nearly zero-pressure gradient in streamwise direction. A sketch of the setup is given in Fig. 1.

A tip vortex is generated by an elliptic planform wing, with a NACA $66_{2}-415$ cross section, and $a=0.8$ mean line. The method for modifying the mean line is described in detail by Abbott and Doenhoff (1959). Due to the manufacturing limitations, the trailing edge was truncated at a thickness of $0.3 \mathrm{~mm}$. This resulted in a chord at the root of the wing of $c_{0}=0.1256 \mathrm{~m}$. The half span of $0.150 \mathrm{~m}$ positions the tip approximately in the center of the test section. The wing was mounted in a six-component force/torque sensor (ATI SI-330-30), in the side window of the test section. At a positive angle of attack, lift points in vertically downward direction.

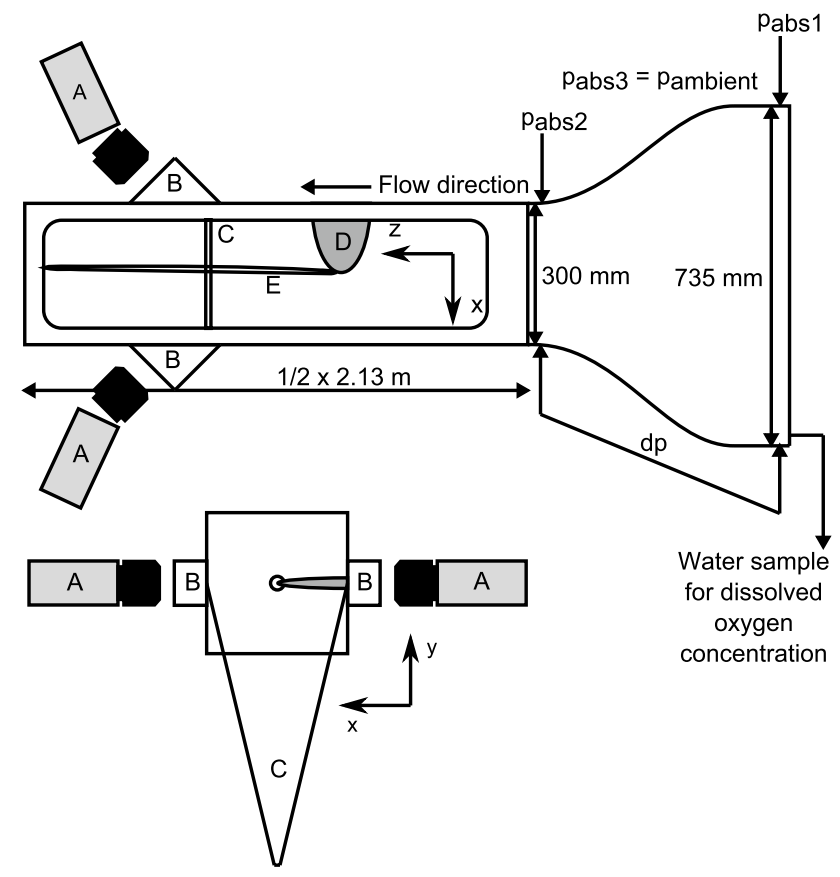

Fig. 1 Experimental setup in the cavitation tunnel with cameras $A$, prisms $B$, laser light sheet $C$, elliptic planform wing $D$ and tip vortex cavity $E$. Location of the absolute and differential pressure sensors is given by $p_{\text {abs }}$ and $d p$, respectively. For the location of the temperature sensor and further tunnel details see Foeth (2008), Zverkhovskyi (2014). The coordinate system origin is at the wing tip. An example of a tip vortex cavity image is given in Fig. 2 
Primary tunnel parameters were measured during each experiment. A temperature sensor (PT-100) was placed submerged in the tunnel water, but outside of the main flow downstream of the test section. Static pressure was measured at $10 \mathrm{~Hz}$ with a digital absolute pressure sensor (Keller PAA 33X), at the outlet of the contraction, upstream of the inlet of the test section, at the vertical position of the wing. The typical accuracy is $0.05 \%$ of full-scale pressure $\left(3 \times 10^{5} \mathrm{~Pa}\right)$, which is $150 \mathrm{~Pa}$. The tunnel free stream velocity, that was also used for the motor control, was based on the pressure drop over the contraction. This is measured with a pressure differential sensor (Validyne DP 15) with a number 36 membrane. The typical accuracy is $0.25 \%$ of full-scale pressure $(35 \mathrm{kPa})$, which is $90 \mathrm{~Pa}$. Both pressure sensor values were corrected with a reference measurement, using a pitot tube at the same location as the wing. All sensors were registered at a frequency of $10 \mathrm{kHz}$.

In the case of cavitation, the dissolved gas concentration partly determines the size of the cavity. Dissolved oxygen (DO) was used here as a representative parameter, determined by using an optical sensor (RDO Pro). Reference measurements were performed with a total dissolved gas sensor. This shows a constant ratio of dissolved oxygen to total dissolved gas. Prior to the experiments, a two-point calibration was performed, using water saturated air (100\% saturation), and fresh sodium sulfite solution (0\% saturation). The sensor was placed in a beaker of tunnel water, which was taken at the start and the end of each series of measurements. The mean of these was used, revealing a small variation of $0.1 \mathrm{mg} / \mathrm{l}$ between the start and the end value, after approximately $3 \mathrm{~h}$.

Two cameras (LaVision Imager Pro LX 16M) were used, with a $90^{\circ}$ spread angle, placed in a horizontal plane near the side windows while viewing upstream. The pixel size of the cameras is $7.4 \mu \mathrm{m}$, at an image format of $3248 \times 4872$ pixels. The cameras with $200 \mathrm{~mm}$ objectives (1:4 D Nikon ED AF Nikkor) were mounted on a Scheimpflug adapter set at an angle of $20^{\circ}$. There are optical aberrations resulting from the stereo viewing through the acrylic test section windows. To partly compensate for this, a $f$-stop of $f / 32$ was used, for a large depth of focus. Due to limitations in the Scheimpflug angle, the placement of the cameras resulted in a magnification of $M_{0}=0.54$. The pixel size in the object plane was $13.8 \mu \mathrm{m}$.

A laser (Spectra-Physics Quanta-Ray PIV-400), at $532 \mathrm{~nm}$ and $350 \mathrm{~mJ}$ per pulse, was used to generate a light sheet. The estimated thickness is below $1 \mathrm{~mm}$. An exposure-time delay between 14 and $6 \mu$ s was used, based on the free stream velocity and the proximity to the wing tip. At a repetition rate of $1 \mathrm{~Hz}, 500$ image pairs were taken as the basis for each measurement.

Stereo calibration was performed by placing a glass plate, with regular dot pattern, perpendicular to the flow, in the test section. A micro-traverse was used to displace the grid in streamwise direction resulting in two calibration planes. The section was accessed by opening the top window, also allowing the target to be submerged during calibration. A calibration was performed after each streamwise plane relocation, and at the start and the end of each day. After calibration, the light sheet was placed on the mid-plane between the calibration planes. This resulted in a very small stereo self-calibration correction.

The flow was seeded with $10 \mu \mathrm{m}$ hollow glass particles (Sphericells). The density of the particles was close to that of water. Even when considering the maximum rotational frequency of the strongest tip vortex, with a typical azimuthal velocity of $7 \mathrm{~m} / \mathrm{s}$ at $1 \mathrm{~mm}$ from the vortex center, the particles still remained high-fidelity flow tracers (Mei 1996). This corresponds to $5 \times 10^{3}$ times the gravitational acceleration, which is challenging for a PIV experiment. The particle images were typically more than 4 pixels in size, thereby circumventing the peak-locking problem (Prasad et al. 1992). The vapor core of vortex cavitation was not seeded, and therefore the velocity was measured only outside the tip vortex cavity.

\section{Particle images, vector processing and high-resolution time-averaging method}

An example of particle images, from both the root-side camera (left) and the tip-side camera (right), is given in Fig. 3. The lift coefficient $C_{\mathrm{L}}$ is defined as $C_{\mathrm{L}}=F_{\mathrm{L}} /\left(\frac{1}{2} \rho W_{\infty}^{2} S\right)$ using the lift force $F_{\mathrm{L}}$, water density $\rho$, free stream velocity $W_{\infty}$ and wing surface area $S=1.465 \times 10^{-2} \mathrm{~m}^{2}$. The Reynolds number $R e=W_{\infty} c_{0} / v$ is based on the free stream velocity $W_{\infty}$, wing root chord $c_{0}$ and kinematic viscosity $\nu$. The cavitation number $\sigma=\left(p-p_{v}\right) /\left(\frac{1}{2} \rho W_{\infty}^{2}\right)$ uses the static pressure $p$ at the test section inlet minus the vapor pressure $p_{v}$. Under these conditions, cavitation is normally not expected. However, a clear white line and faint shadow were observed in the top half of the image. The seeding particles act as nucleation sites and promote cavitation inception. The intermittent streak of cavitation in the vortex core was visible as a white line. This was absent under the same conditions without seeding. Since the diameter was very small, it is not expected to strongly influence the flow field. It does deteriorate the quality of the vectors along this bright white line.

In the case of a large tip vortex cavity, the PIV images look similar to those in Fig. 4. The cavity can be distinguished by a bright underside and a distinct vertical shadow. It is clear from these images that no meaningful velocity information can be obtained from the region blocked by the image of the cavity. The particles in the shadow might still be useful, though the lower intensity will affect the vector quality at the edges of the shadow. 


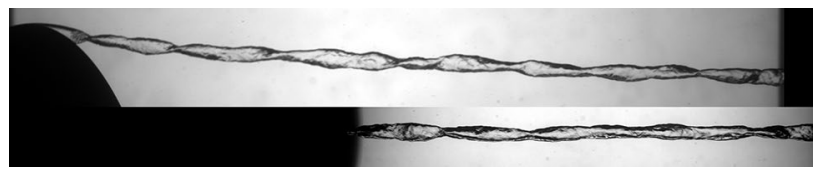

Fig. 2 Example of high-speed video images reproduced from Pennings et al. (2015). Flow is from left to right. In the present study, the top and bottom images correspond to the $x z$-plane and the $y z$ plane, respectively. The pixel size in the object domain was $16 \mu \mathrm{m}$ in the $x z$-plane, and $10 \mu \mathrm{m}$ in the $y x$-plane. Black object on the top is the pressure side of the wing. Conditions: lift coefficient $C_{\mathrm{L}}=0.58$, Reynolds number $R e=9.1 \times 10^{5}$ and dissolved oxygen concentration $\mathrm{DO}=2.7 \mathrm{mg} / \mathrm{l}$

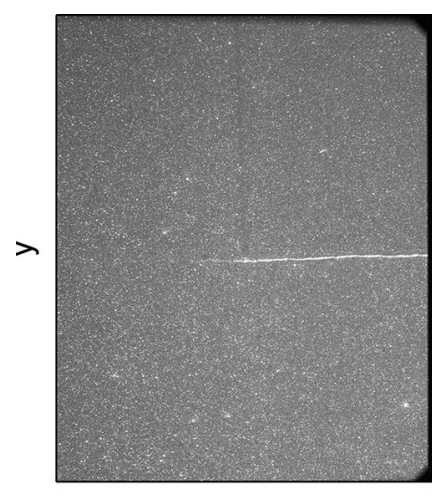

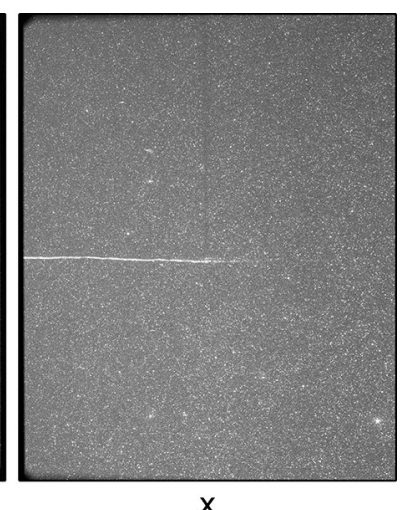

Fig. 3 Particle images of both cameras. Conditions: $z / c_{0}=1.14$, $C_{\mathrm{L}}=0.65, \operatorname{Re}=8.9 \times 10^{5}, \sigma=4.2$ and $\mathrm{DO}=2.5 \mathrm{mg} / \mathrm{l}$
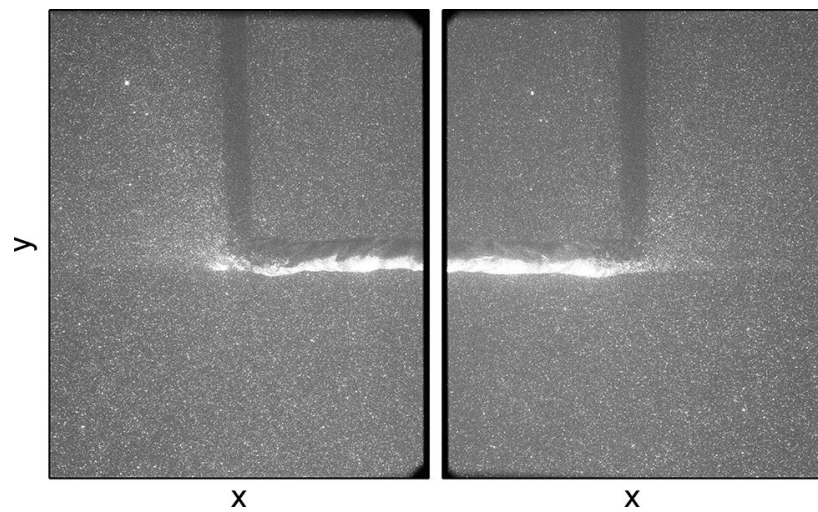

Fig. 4 Particle images of both cameras. Conditions: $z / c_{0}=1.14$, $C_{\mathrm{L}}=0.67, \operatorname{Re}=9.0 \times 10^{5}, \sigma=1.1$ and $\mathrm{DO}=2.5 \mathrm{mg} / \mathrm{l}$

The light sheet was refracted and reflected on the cavity interface. The reflections were of low enough brightness not to pose a significant risk of damaging the cameras. However, these effects create shadows and local bright regions in the particle images. An alternative would be to use fluorescent tracer particles. This requires a significant increase in the light required to illuminate a moderately sized field of view. The regular tracer particles already required the maximum laser pulse energy. The compromise then was to take a $90^{\circ}$ wedge (shown in Fig. 5). Vectors inside the cavity interface were excluded from analysis.

The seeding density is limited by the distance, which is half the tunnel height, that the light sheet has to travel before it illuminates the field of view. Also, particle image pairs are lost due to a large out of plane velocity. These parameters result in instantaneous vector fields of insufficient quality. Since the conditions during the typical $10 \mathrm{~min}$ measurement were stable, the vector fields could be averaged. The result of a simple average is shown in Fig. 5. Even under stable conditions, a tip vortex center position in the proximity of the tunnel walls showed displacement or wandering. Figure 5 also shows the result of two stages of improvement. The first stage involved conditional averaging of the vortex center position. The vortex center was obtained by summing the absolute values of the vertical and horizontal velocity components, and fitting a parabola through the highest values, to obtain the coordinates of the maximum. In the next stage, these vortex centers were used to reprocess the particle images using sum of correlation (SOC), on images with similar vortex center positions. The details of these approaches are described at the end of this section.

Vector fields were obtained using DaVis 8. The images were preprocessed with a $96 \times 96$ pixel sliding background filter. A geometric mask was applied to exclude the image edges from vector calculation. Stereo cross correlation was applied using a multi-pass approach with 3 passes on $64 \times 64$ pixel areas with $50 \%$ overlap, and 2 passes on $48 \times 48$ pixel areas with $50 \%$ overlap. This resulted in a vector spacing of $0.3 \mathrm{~mm}$. During post-processing, a minimum peak ratio of 1.5 was imposed, and a median test was applied for outlier detection.

The global vortex detection method by Graftieaux et al. (2001) and used in Dreyer et al. (2014) was also considered. This method is much more robust due to lower susceptibility to noise in the instantaneous vector fields. However, the bright line in the particle images as seen in Figs. 3 and 4 resulted in low vector quality. Therefore the method could not determine the horizontal position with good accuracy. This region does not provide meaningful information and further increases in size at proper tip vortex cavitation. Therefore the global vortex detection method could not be used to estimate the horizontal position of the vortex center.

The instantaneous particle images of Fig. 4 were therefore used. First, the bright underside of the cavity was detected. This was used as a reference for the vertical center position. From this location downward, the horizontal velocity was used. In a similar manner for non-cavitating cases the horizontal position of the center was thus obtained. The original particle images were shifted to the 

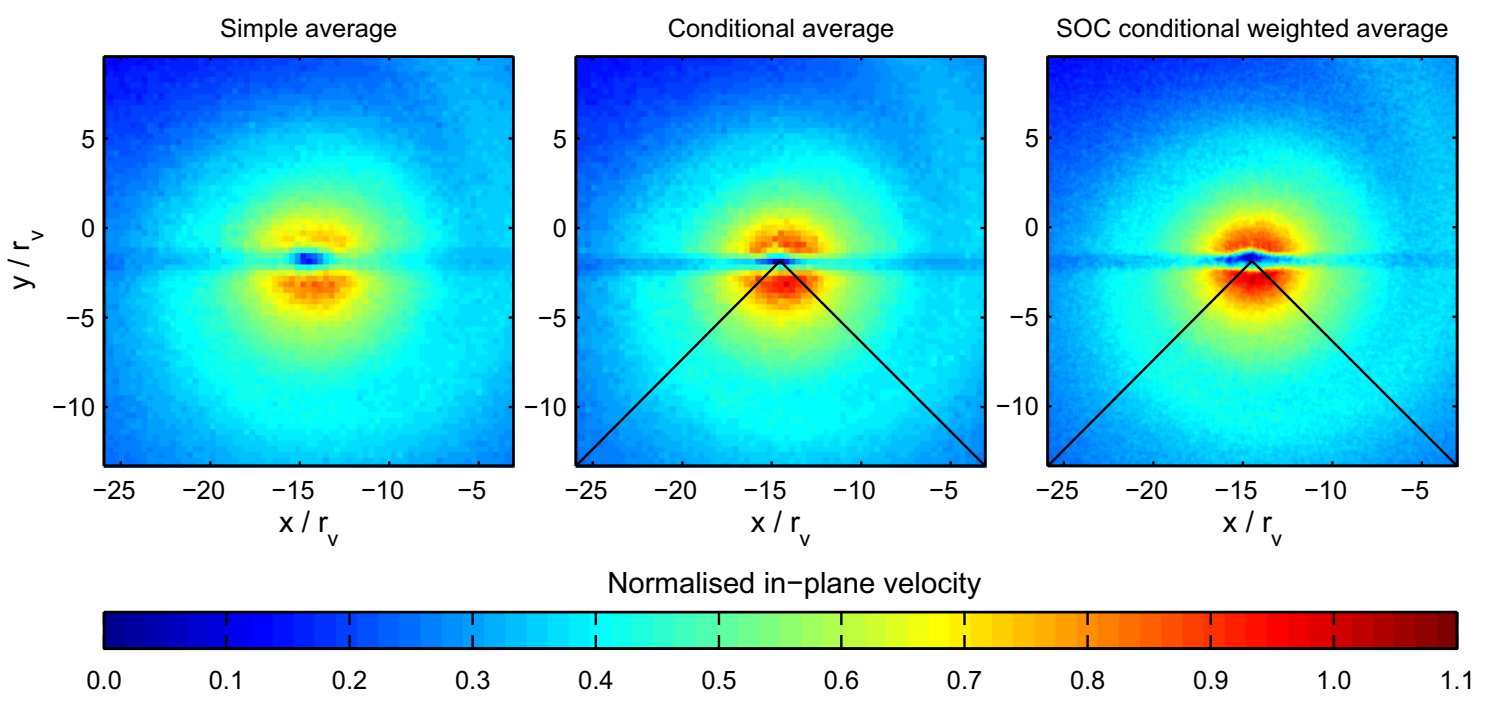

Fig. 5 Normal time average, conditional vortex centered average and sum of correlation (SOC) conditional weighted average of the in-plane velocity normalized with the free stream velocity $W_{\infty}=6.8 \mathrm{~m} / \mathrm{s}$ and viscous core radius $r_{v}=1.1 \mathrm{~mm}$. Black lines indi-

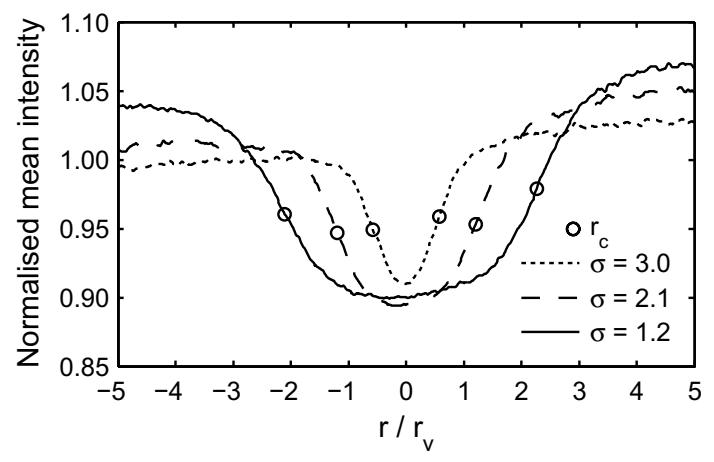

Fig. 6 Normalized mean intensities of the vortex cavity shadow, with the open circles indicating the location of maximum gradient set as the cavity edge radius $r_{c}$, normalized with wetted viscous core radius $r_{v}=1.1 \mathrm{~mm}$. Conditions: $z / c_{0}=1.14, C_{\mathrm{L}}=0.66, R e=9.1 \times 10^{5}$ and $\mathrm{DO}=2.5 \mathrm{mg} / \mathrm{l}$

vortex center and summed. In this summation, the vertical shadow was used to obtain the cavity diameter. The result is shown in Fig. 6. The condition for the cavity edge was taken to be the location at which the derivative of the intensity is maximum.

In the results section, this method is compared to the shadowgraphy high-speed video of Pennings et al. (2015). An example image of two views is given in Fig. 2. The largest variability on the mean cavity diameter corresponds to the amplitude of the stationary wave shape. This mainly affects the cavities with a diameter of several times the wetted viscous core radius. cate the $90^{\circ}$ section of the velocity field, that is later used for the contour average. Conditions: $z / c_{0}=1.14, C_{\mathrm{L}}=0.65, R e=8.9 \times 10^{5}$, $\sigma=4.2$ and $\mathrm{DO}=2.6 \mathrm{mg} / \mathrm{l}$

The vortex center was reliably detected in the instantaneous vector fields. This results in a residual vortex motion equal to the vector spacing of $0.3 \mathrm{~mm}$. This vector spacing is reasonable for global properties but is insufficient to capture the detailed dynamics near the edge of the viscous vortex core, or in the case of cavitation close to the vaporfilled cavity edge.

The vector spacing in the present study was limited by the number of well-correlated particle image pairs in an interrogation area. The practical limit of sufficient particle image pairs for each time instance was reached at $48 \times 48$ pixels areas.

Since the vortex center positions were known, particle images with the vortex at the same location were selected and processed together. The correlation maps of all the individual instantaneous particle images, at the same vortex center position, were summed to obtain a single vector field. This is based on the correlation averaging method by Meinhart et al. (2000), referred to here by SOC. In this manner, the number of well-correlated particle image pairs increases with the number of images used. Meinhart et al. (2000) also concluded that the correlation averaging technique results in improvement of vector correlation quality while allowing reduction in the interrogation area size.

For all streamwise locations except $z / c_{0}=5.50$, the interrogation area size was reduced to values in Table 1 . This shows the minimum area size, to be able to ensure at least $95 \%$ good vectors, at the vortex center positions, with the least number of available images. To benefit from this 
Table 1 Properties for SOC processing

\begin{tabular}{llllll}
\hline$z / c_{0}$ & 0.50 & 0.74 & 1.14 & 1.75 & 5.50 \\
Minimum number of images per vortex center position & 38 & 22 & 13 & 8 & - \\
Average number of vortex center positions & 5 & 8 & 14 & 22 & - \\
Interrogation area (pixels) & $6 \times 6$ & $8 \times 8$ & $12 \times 12$ & $24 \times 24$ & $48 \times 48$ \\
Vector spacing $(\mu \mathrm{m})$ & 42 & 55 & 83 & 165 & 332 \\
\hline
\end{tabular}

Due to insufficient number of images SOC is not applied at $z / c_{0}=5.50$. The results at that location are based on the conditional average approach, a certain minimum number of images on a single vortex center position is needed. The minimum number of images used in the SOC for each streamwise location is given in Table 1. The number of vortex center positions is chosen such that an approximately equal total number of images is used for all cases. At $z / c_{0}=5.50$, the vortex center wandering amplitude is so large, that this minimum condition could not be met at an improved interrogation area size.

The vector calculation for the SOC approach is also based on multi-pass iterations. First, 3 passes on $32 \times 32$ pixels areas using $50 \%$ overlap were followed by 2 passes at the area size given in Table 1 also with a $50 \%$ overlap. A minimum peak ratio of 1.5 was required for the three passes of a universal-outlier detection with a $5 \times 5$ vectors filter region. Empty spaces in the vector field were interpolated.

The result of SOC on the various positions could be considered as new 'instantaneous' vector fields. The new SOC vector fields were then displaced to match the vortex center position. The final averaged SOC vector field was obtained by a weighted average based on the number of images used in the individual SOC vector fields. A sample of this approach is given in Fig. 7. This results in a dimensional vector spacing as given in Table 1 , which is $2-8$ times smaller than the original interrogation area size. The following results are based on vector processing using SOC except for $z / c_{0}=5.50$, which is based on vortex center conditional averaging.

\section{Results}

The results are presented as follows. A general overview of the properties of a wetted vortex flow field is given in Sect. 4.1. This includes the residual error of the vortex center motion, the optical aberrations in several streamwise locations and the empirical model fit. In Sect. 4.2, the wetted flow field is compared to the one around the vortex cavity. Finally, in Sect. 4.3, the obtained cavity angular velocity is used to calculate the tip vortex cavity resonance frequency.

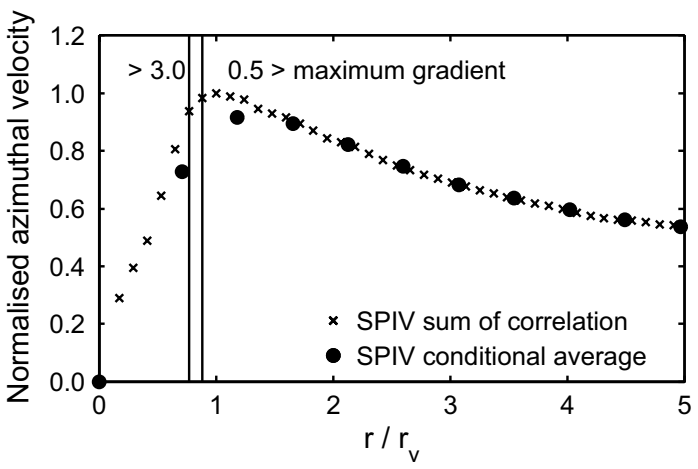

Fig. 7 Result of SOC method on contour-averaged data, normalized with the maximum azimuthal velocity $u_{\theta}=-6.7 \mathrm{~m} / \mathrm{s}$ and the viscous core radius $r_{v}=1.1 \mathrm{~mm}$. Conditions: $z / c_{0}=1.14, C_{\mathrm{L}}=0.65$, $R e=8.9 \times 10^{5}, \sigma=4.2$ and $\mathrm{DO}=2.5 \mathrm{mg} / \mathrm{l}$. The original conditionally averaged data are also given, to show the improvement to the description of the azimuthal velocity around the viscous core. The vertical lines indicate the limits where the gradient is $>3.0$ and $<0.5$ pixel per $48 \times 48$ pixels interrogation area. Left of the $>3.0$ pixel line is not properly resolved by the conditional average data (Westerweel 2008). The allowable gradient for the SOC data is higher than the measured viscous core gradient

\subsection{Wetted vortex flow field}

The lift coefficient $C_{\mathrm{L}}$ is used throughout this study to indicate the condition under which the measurements were performed. This gives an indication of the strength of the tip vortex. Figure 8 shows the relation between the angle of attack $\alpha$ and the lift coefficient. At cavitation numbers below 4 , a steady tip vortex cavity was present. This effect on the lift coefficient falls below the amplitude of the signal variability, approximately equal to the repeatability error in setting the angle of attack. The drag force was an order of magnitude smaller than the lift force. Since this is not accurately resolved by the force sensor and was irrelevant for the present study, it is not discussed further.

\subsubsection{Vortex wandering statistics}

The vortex center position can be used to describe the characteristics of the motion in two directions. The symbols 


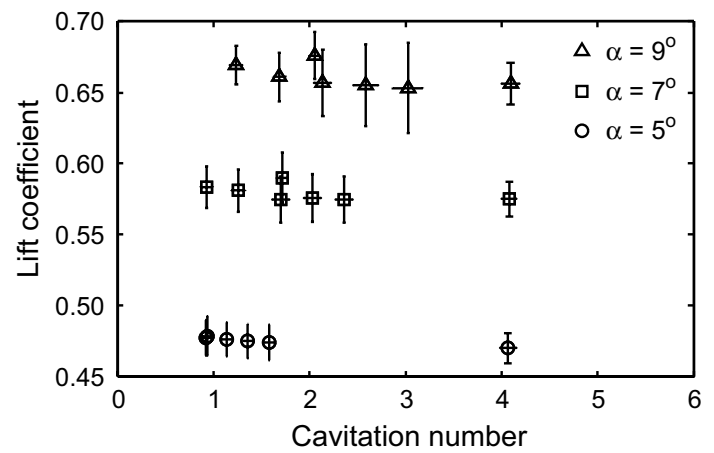

Fig. 8 Effect of cavitation number on lift coefficient for three angles of attack $(\alpha)$ in degrees. Conditions: $R e=9 \times 10^{5}$ and $\mathrm{DO}=2.5 \mathrm{mg} / \mathrm{l}$

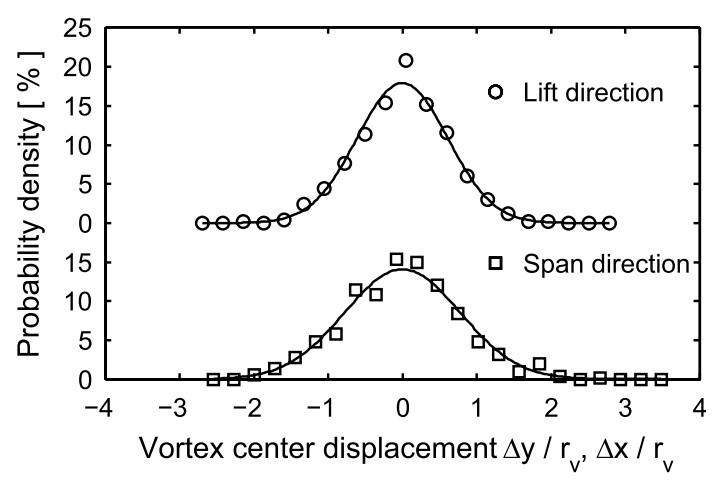

Fig. 9 Probability density of vortex center displacement, normalized with viscous core radius $r_{v}=1.2 \mathrm{~mm}$. Lines are normal distributions based on standard deviations of vortex center location. Conditions: $z / c_{0}=1.75, C_{\mathrm{L}}=0.66, R e=9.1 \times 10^{5}, \sigma=4.1$ and $\mathrm{DO}=2.6 \mathrm{mg} / 1$

in Fig. 9 are obtained from a histogram of the measured vortex centers, with each bin equal to one vector spacing. The lines are normal distributions, based on the standard deviation of the vortex center motion. Figure 10 shows the relation between vortex center motion and streamwise position.

In general, the vortex center motion was larger in the spanwise direction than in the lift direction. The only exception found was at $C_{\mathrm{L}}=0.66$ with $z / c_{0}=5.50$. Except for this particular case, the amplitude of motion was hardly influenced by the lift coefficient. The motion amplitude increased in proportion to downstream distance.

Vector spacing limited the accuracy of determining the vortex center position. The residual motion was smeared out in the SOC approach. As studied by Devenport et al. (1996), this residual motion was analytically estimated with good accuracy, as found by Bhagwat and Ramasamy (2012). For a laminar $q$-vortex, as described by Batchelor (1964), the ratio of the real viscous core size to the measured viscous core size is defined as:

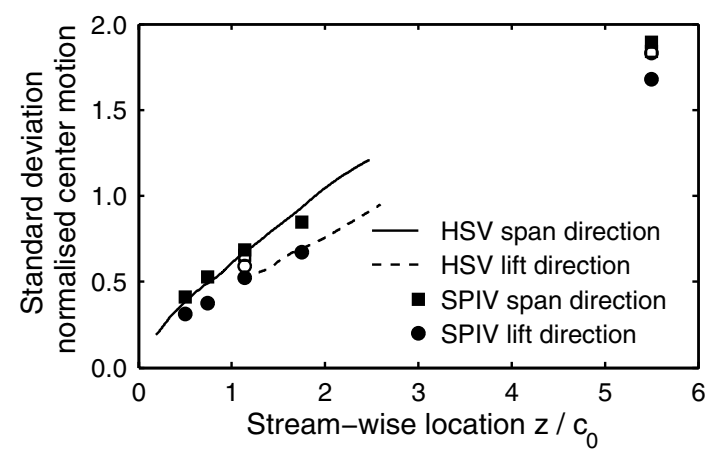

Fig. 10 Standard deviation of vortex center motion, normalized with mean wetted viscous core size $r_{v}=1.0 \mathrm{~mm}$. The filled symbols are for flow without cavitation. The open symbols are for $\sigma=1.26$ at $z / c_{0}=1.14$ and $\sigma=1.72$ at $z / c_{0}=5.50$. The high-speed video (HSV) data is taken from Pennings et al. (2015) at $\sigma=1.20$. Conditions: $C_{\mathrm{L}}=0.58, R e=9.3 \times 10^{5}, \sigma=4.1$ and $\mathrm{DO}=2.6 \mathrm{mg} / \mathrm{l}$

$\frac{r_{v(\text { real })}}{r_{v \text { (measured })}}=\sqrt{1-\frac{2 a s^{2}}{r_{v(\text { measured })}^{2}}}$,

where $a=1.25643$, and $s$ is the residual motion standard deviation of the vortex center motion, within one vector spacing of $s=0.33 \mathrm{~mm}$. This value was 0.289 vector spacing for all cases or $0.289 \times 0.33 \mathrm{~mm}=96 \mu \mathrm{m}$. For the measured viscous core size $r_{v \text { (measured), the estimates }}$ of Fig. 13 were used. This caused a maximum overestimation of the viscous core size and subsequent underestimation of the azimuthal peak velocity of $2 \%$ for cases at $z / c_{0}=0.50$. Typical residual errors for the other streamwise locations were $1 \%$ or lower and were therefore not considered further.

\subsubsection{Streamwise development}

An overview of the development of the streamwise and inplane velocity is given in Fig. 11 . The case at $C_{\mathrm{L}}=0.66$ without cavitation was chosen, because it shows the highest velocities and strongest gradients. The origin of the coordinate system is the upstream wing tip. The horizontal axis selection was chosen around the center of the tip vortex. The vortex center is seen to move horizontally toward the root. Some features that reduced the quality of the results are described here.

The horizontal line through the velocity fields is related to the line in Fig. 3. At $z / c_{0}=0.50$ and $z / c_{0}=1.75$, vertical lines were also present. This was due to the seal of the water-filled prism. The prism was open on the side mounted to the test section windows. The edges were sealed using an o-ring. The results at five streamwise locations were obtained by moving the entire SPIV system comprised of prisms, cameras and light sheet. The impression of the 
Fig. 11 Streamwise development of axial and in-plane velocity, normalized with free stream velocity $W_{\infty}=6.8 \mathrm{~m} / \mathrm{s}$ and mean viscous core size $r_{v}=1.1 \mathrm{~mm}$. Conditions: $C_{\mathrm{L}}=0.66, R e=9.2 \times 10^{5}$, $\sigma=4.1$ and $\mathrm{DO}=2.5 \mathrm{mg} / \mathrm{l}$

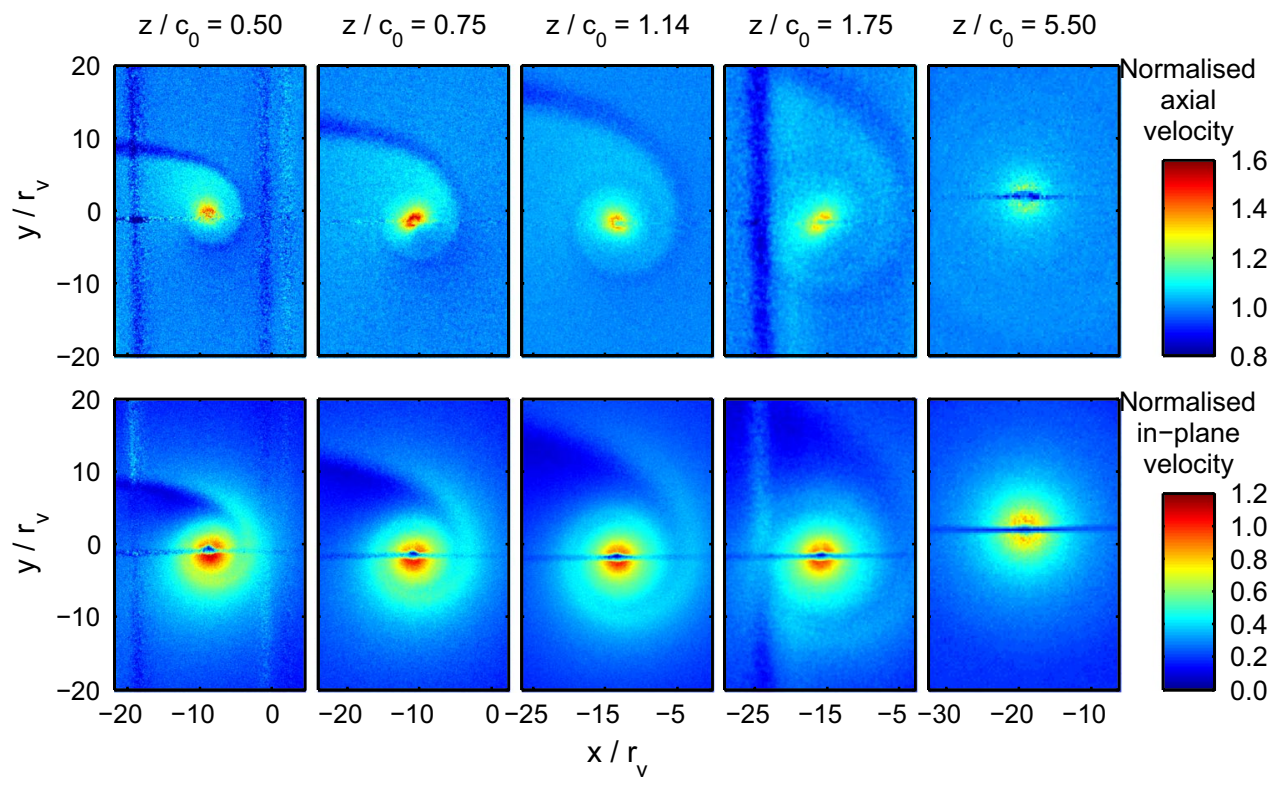

seal caused a deformation in the acrylic test section window, which was visible in the particle images and introduced errors in the vector calculations. Due to the order of the measurements, these vertical lines were absent at $z / c_{0}=0.75, z / c_{0}=1.14$ and $z / c_{0}=5.50$. These features as shown in Fig. 11 reduced the vector quality and limited the useful area for comparison. In case of cavitation, the upper central part was in the cavity shadow and is therefore not useful.

Since the wing was in a pitch-up position, with the suction side at the bottom, the trailing edge was located at positive vertical values. The wake of the wing, in the upper streamwise or axial velocities, appear as a dark blue spiral. At $z / c_{0}=0.75$ the axial velocities in the vortex core were highest, which imposes a challenge for the SPIV method used here, due to the out of plane loss of particles. This was resolved by choosing a smaller time delay between exposures. This reduced the in-plane particle displacement, which reduces the vector calculation accuracy. The height of the correlation peak was finally increased by using correlation averaging. The axial velocity obtained from SPIV, at the outer edge of the field of view, was within $1 \%$ of the velocity obtained from the contraction pressure drop. Downstream the effect of the roll-up of the wing wake reduces this axial velocity excess.

A reference part of the plane was chosen as a wedge from the vortex center downward defined by a $90^{\circ}$ angle. This wedge captures the highest velocities and is not hindered by the detrimental features such as the cavity shadow, cavity reflections and the vertical window deformation due to the prisms. An example of this region is presented in Fig. 5. In the case of the vertical distortions, the extent of the wedge in $x$-direction was limited. Using the identified vortex center, a polar coordinate system was used and the data were averaged over contours with constant radius.

\subsubsection{Empirical vortex model parameter estimation}

Most common viscous axisymmetric vortex models are collectively described by $\mathrm{Wu}$ et al. (2006) [such as the simple Rankine vortex, the families of Gaussian vortex models such as the Lamb-Oseen, Burgers and Batchelor (1964) vortex models or the empirical Burnham and Hallock (1982) vortex model]. For these models, two parameters are sufficient to describe the velocity field. These are usually the viscous core radius $r_{v}$ and the vortex circulation $\Gamma$. In all of the above-mentioned models, the vorticity was strongly concentrated close to the vortex center. As can be seen in Fig. 11, the tip vortex is still in the process of rollup and includes the wing boundary layer wake. The combination of a small viscous core, a reduced peak azimuthal velocity due to the wake, and a larger spread of the vorticity deems all of the above-mentioned models unsuitable for fitting to the data of the present study.

The Winckelmans (Gerz et al. 2005) model and the Proctor (1998) model are two closely related empirical vortex models that include extra parameters to better match the contour-averaged data.

The Winckelmans vortex model was used to obtain a complete description of the measurement data, using a large number of fit parameters. The Winckelmans model is the same as the Proctor model outside $r>1.15 r_{v}$. Both models failed to accurately describe the cavity size within the wetted viscous core radius. The small viscous core size presented a model-scale issue and was not relevant for the vortex cavity resonance frequency on full-scale propellers. 
Therefore, inclusion of only the part outside the wetted viscous core in the Proctor model reduced the number of parameters.

The azimuthal velocity $u_{\theta}$ is defined as:

$$
\begin{aligned}
u_{\theta}= & \frac{\Gamma}{2 \pi r} \\
& \left(1-\exp \left(\frac{-\beta_{\mathrm{i}}\left(\frac{r}{B}\right)^{2}}{\left(1+\left(\frac{\beta_{\mathrm{i}}}{\beta_{\mathrm{o}}}\left(\frac{r}{B}\right)^{5 / 4}\right)^{p}\right)^{1 / p}}\right)\right),
\end{aligned}
$$

where $\Gamma$ is the vortex circulation. The value that gave a good description of the flow field was defined as $\Gamma=\frac{1}{2} c_{0} C_{\mathrm{L}} W_{\infty} \pi / 4$. The total wing span $B$ was $0.30 \mathrm{~m}$. Three free parameters remain to be fitted to the experimental data. The outer scale $\beta_{\mathrm{o}}$ can account for the vortex roll-up and inclusion of the wing boundary layer wake in the outer part of the vortex flow. The inner scale $\beta_{\mathrm{i}}$ sets the approximate relation for the viscous core as $r_{v} / B \approx\left(\beta_{\mathrm{o}} / \beta_{\mathrm{i}}\right)^{4 / 5}$. The value for $p$ was used to match the peak velocity at the viscous core. The Winckelmans model was not intended for the description of the flow around vortex cavitation, but it appears quite capable to do so. The result of the model fit to the experimental data is given in Fig. 12.

All parameters revealed large changes in the velocity field close to the wing tip. The Winckelmans model is intended for well-developed airplane wing tip vortices. The variability of the $p$ parameter might be an indication that the model is used outside the conventional range. All values above 4 indicate much higher peak velocities than commonly found in the airplane wing tip vortices. This also applies to the high values found for $\beta_{\mathrm{i}}$. The $\beta_{\mathrm{o}}$ value is strongly related to the azimuthal velocity close to the vortex core and is most dependent on the lift coefficient. The roll-up of the tip vortex is related to the loading distribution on the wing. Higher lift coefficients have a larger gradient in loading close to the tip. This results in a faster roll-up and higher $\beta_{\mathrm{o}}$ value.

The Winckelmans model fit gives an accurate estimation of the viscous core size of the vortex, which is the radial location of maximum azimuthal velocity. The development of the viscous core size for streamwise location and lift coefficient is given in Fig. 13 and is made dimensionless using an equivalent turbulent boundary layer thickness taken from Astolfi et al. (1999) as $\delta=0.37 c_{0} R e^{-0.2}$.

The values of $r_{v} / \delta$ in the present study are significantly smaller than those reported by Astolfi et al. (1999). They found, between streamwise locations $z / c_{0}=0.5$ to 1.0 , values between $r_{v} / \delta=0.8$ and 1.1. These differences could be due to very high spatial resolution, small measurement volume, accurate vortex wandering removal due to SOCweighted conditional averaging and a cross-sectional wing
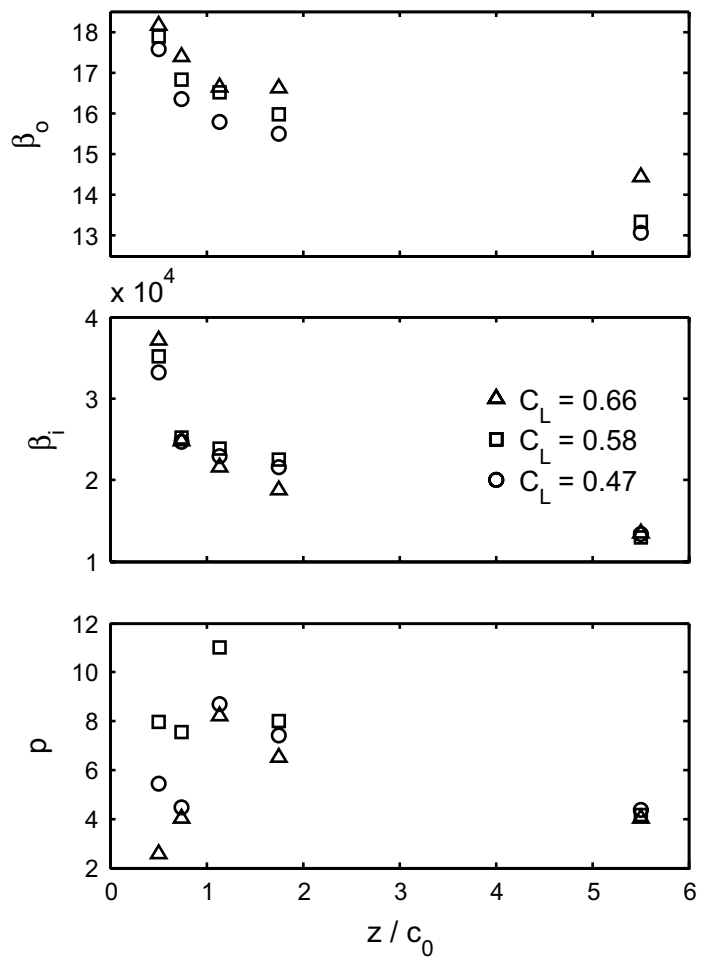

Fig. 12 Parameters of Winckelmans vortex model fit to contour-averaged data at conditions: $R e=9.3 \times 10^{5}, \sigma=4.1$ and $\mathrm{DO}=2.5 \mathrm{mg} / \mathrm{l}$

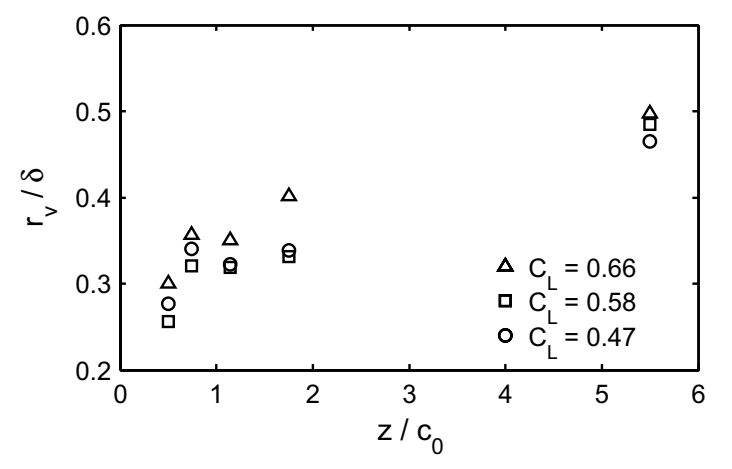

Fig. 13 Dimensionless viscous core size obtained from location of maximum azimuthal velocity of Winckelmans vortex model fit of Fig. 12

geometry designed for a large laminar boundary layer extent in the present study.

The results of $r_{v}$ was used to select part of the flow that is outside $r \geq 1.15 r_{v}$. This part was fitted using the simpler adapted Proctor (1998) vortex model. The azimuthal velocity $u_{\theta}$ is defined as:

$u_{\theta}=\frac{\Gamma}{2 \pi r}\left(1-\exp \left(-\beta\left(\frac{r}{B}\right)^{0.75}\right)\right)$, 


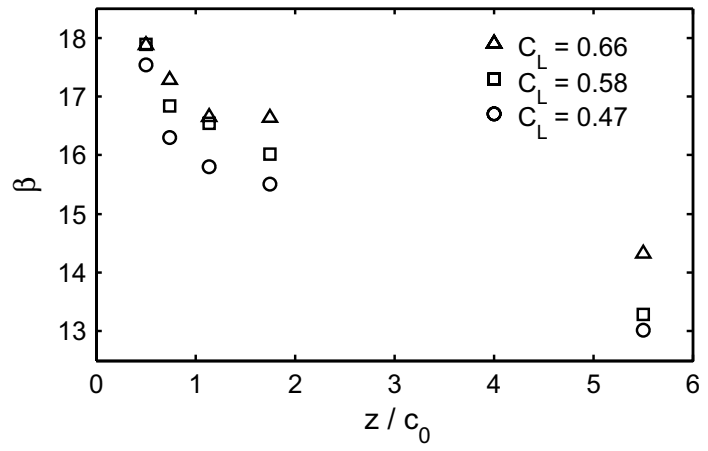

Fig. 14 Parameter of Proctor vortex model fit to contour-averaged data at conditions: $R e=9.3 \times 10^{5}, \sigma=4.1$ and $\mathrm{DO}=2.5 \mathrm{mg} / \mathrm{l}$

where the parameters $\Gamma$ and $B$ are equal to the Winckelmans model. The value for $\beta$ could be taken equal to $\beta_{\mathrm{o}}$, but for consistency the model is fitted directly to the experimental data. As the Winckelmans model is intentionally similar to the Proctor model, outside the vortex viscous core, the values for $\beta$ and $\beta_{\mathrm{o}}$ were found to be close. As an engineering model, the Proctor model is preferred, as it needs only one fitting parameter and requires no prior knowledge of the viscous core size. The fit values of the Proctor vortex model are given in Fig. 14.

The Proctor vortex model is used to estimate the cavity size in the case of cavitation. Assuming axisymmetry and zero radial velocity, the conservation of radial momentum equation simplifies to:

$\frac{\mathrm{d} p}{\mathrm{~d} r}=\rho \frac{u_{\theta}^{2}}{r}$.

At the tunnel wall, approximately $0.15 \mathrm{~m}$ from the vortex center, the pressure was assumed to be the free stream static pressure $p_{\infty}$. By numerical integration of Eq. 4 , the pressure distribution over the radius could be obtained. The cavity radius is defined as the location at which the cavitation number is equal to minus the pressure coefficient $\sigma=-\left(p-p_{\infty}\right) /\left(\frac{1}{2} \rho W_{\infty}^{2}\right)$

\subsection{Comparison between wetted and cavitating vortex}

The experimental vortex cavity size was obtained using two methods. The first method was based on the maximum gradient of cavity shadow in the light sheet shown in Fig. 6. The second method was the Proctor vortex model fit and Eq. 4, to determine the location of vapor pressure and thus the cavity radius. The cavity size based on these methods were compared to the previously obtained cavity size based on high-speed video recordings and is shown in Fig. 15.

Both these methods showed similar trends. There was a good agreement between the SPIV and HSV data. This verified the combined steps, of vortex center localization and

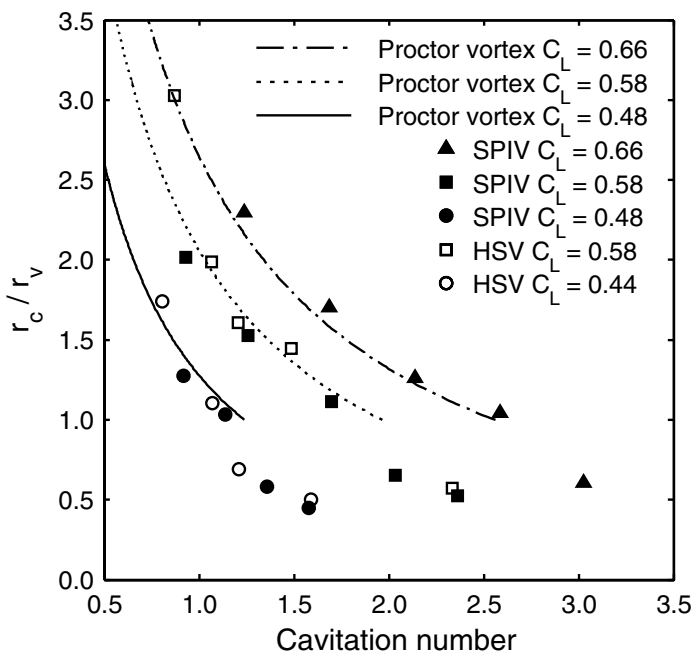

Fig. 15 Cavity radius normalized with wetted viscous core radius $r_{v}=1 \mathrm{~mm}$, as determined from SPIV image shadow, compared with HSV data from Pennings et al. (2015). Conditions: $z / c_{0}=1.14$, $R e=9.2 \times 10^{5}$ and $D O=2.5 \mathrm{mg} / \mathrm{l}$. Proctor vortex model based on values from Fig. 14, which is specifically restricted to outside the viscous core to reduce the number of model parameters. The break in the experimental cavity size trend, inside the viscous core, is poorly described by either empirical vortex model

the use of the maximum intensity gradient in the shadow, for cavity diameter estimation. The edge of the wetted flow field in all following results was based on this post-processing method.

The Proctor vortex model fit gave a good result up to the viscous core. The Winckelmans vortex model describes the inner vortex core velocity well, but overestimated the cavity size if smaller than the viscous core size.

With the cavity size known for all cases, the velocity field outside the cavity was compared to a wetted vortex in Figs. 16 and 17. The lowest three cavitation numbers represented varying cavity sizes. The standard deviation over constant radius contours was given vertically. The standard deviation of cavity diameter was given horizontally at the cavity interface radius.

All the cases with a vapor cavity core showed a region of significantly retarded flow with respect to the flow field without cavitation. Analogous to a wetted viscous vortex core, the velocity gradient in the layer close to the cavity interface approximates solid body rotation. For cavities with $r_{c}>3 r_{v}$, the flow field was approximately equal to the wetted flow field.

\subsection{Tip vortex cavity resonance frequency}

The analysis of HSV data, as shown in Fig. 2, in the wave number and frequency domain resulted in clear dispersion relations of waves traveling on the interface of the vortex 


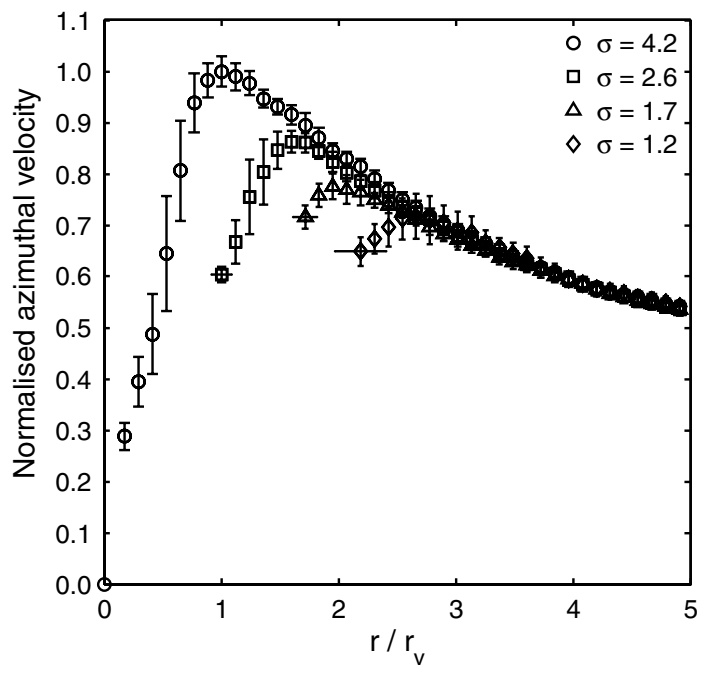

Fig. 16 Comparison between wetted and cavitating vortex SPIV contour-averaged azimuthal velocity, normalized with the maximum wetted azimuthal velocity $u_{\theta}=-6.7 \mathrm{~m} / \mathrm{s}$. The radius is normalized with the wetted viscous core radius $r_{v}=1.1 \mathrm{~mm}$. Conditions: $z / c_{0}=1.14$, $C_{\mathrm{L}}=0.66, R e=9.0 \times 10^{5}$ and $\mathrm{DO}=2.5 \mathrm{mg} / \mathrm{l}$

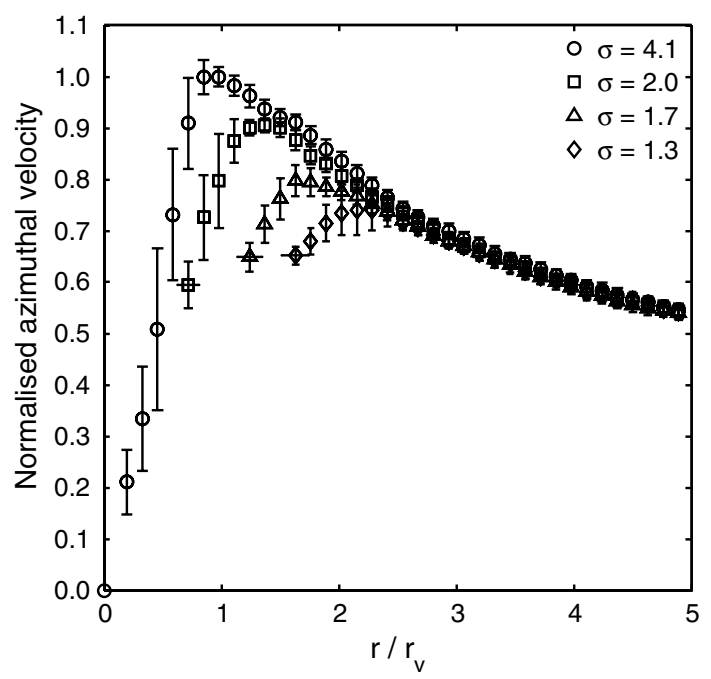

Fig. 17 Comparison between wetted and cavitating vortex SPIV contour-averaged azimuthal velocity, normalized with the maximum wetted azimuthal velocity $u_{\theta}=-6.1 \mathrm{~m} / \mathrm{s}$. The radius is normalized with the wetted viscous core radius $r_{v}=1.0 \mathrm{~mm}$. Conditions: $z / c_{0}=1.14$, $C_{\mathrm{L}}=0.58, R e=9.3 \times 10^{5}$ and $\mathrm{DO}=2.6 \mathrm{mg} / \mathrm{l}$

cavity (Pennings et al. 2015). The dispersion relations of the three dominant deformation modes were described by a model based on a two-dimensional potential flow vortex in uniform axial flow. It was shown to be valid for a viscous vortex to first-order approximation. The model requires four input parameters: the speed of sound in water, the axial flow velocity, the cavity radius and the cavity angular velocity. The cavity angular velocity, which was the only unknown parameter in that study, was obtained by matching the model dispersion relations to experimental results.

Tip vortex cavity resonance frequencies were found directly from the cavity diameter oscillations in the frequency domain. These frequencies are accurately described by a zero group velocity condition on the dispersion relation of the $n=0^{-}$, volume variation mode. Using the cavity angular velocity, found from the match of the model dispersion relations to the experiment, these frequencies are accurately described by the model. The measurements around the vortex cavity in the present study provide the opportunity to verify the validity of the vortex cavity wave dynamics model, via direct measurement of the cavity angular velocity.

The angular velocities at the cavity interface in Figs. 16 and 17 were normalized with the wetted angular velocities at equal radius. The results are presented in Fig. 18. As reference, the values of the cavity angular velocity obtained from the model fit of Pennings et al. (2015) are included.

As already observed in Figs. 16 and 17, the SPIV values were lower than the wetted vortex reference. Strong correlation was found between the HSV results and the wetted vortex velocity field. Its implication can be better appreciated by using the derived values to calculate the tip vortex cavity resonance frequency, in Fig. 19.

The open symbols are resonance frequencies directly obtained from HSV without any intermediate model. The closed symbols are based on direct measurement of the

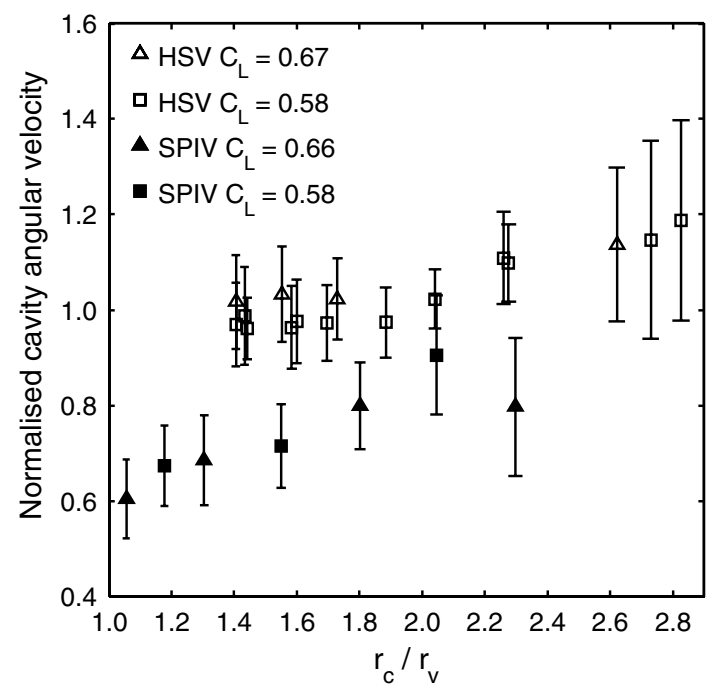

Fig. 18 Cavity angular velocity as a function of cavity radius, normalized with wetted SPIV measurements at $z / c_{0}=1.14$ and viscous core radius $r_{v}=1 \mathrm{~mm}$. Filled symbols are obtained directly from data of vortex cavitation cases in Figs. 16 and 17 at the cavity edge. Open symbols are based on model fit values from Pennings et al. (2015). Range of cavitation number $\sigma=2.8-0.9$ 


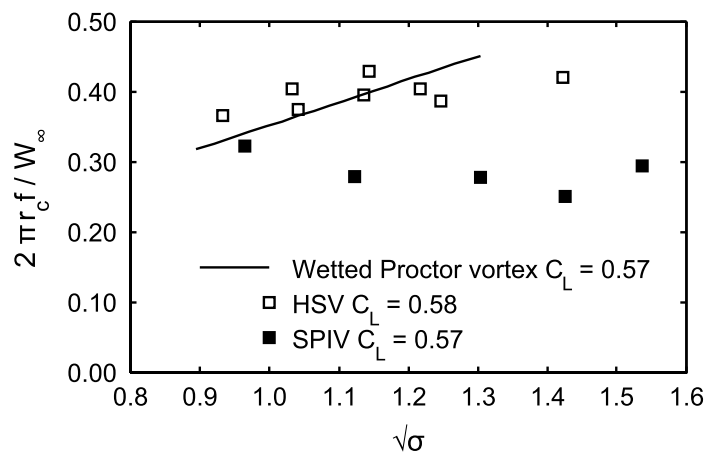

Fig. 19 Non-dimensional cavity resonance frequency as a function of square root of cavitation number. HSV resonance frequencies directly obtained from measurement. SPIV resonance frequencies based on resonance frequency model using measured cavity radius and cavity angular velocity. Wetted Proctor model based on resonance frequency model using wetted angular velocity at cavity radius as model input for the cavity angular velocity. Proctor model parameters: $\Gamma=0.18 \mathrm{~m}^{2} / \mathrm{s}, \beta=16$ and $B=0.3 \mathrm{~m}$. HSV results and model from Pennings et al. (2015)

cavity angular velocity in the present study. Clearly the model for the tip vortex cavity resonance frequency is not physically correct. Based on the high correlation of the HSV values to the wetted vortex reference in Fig. 18, a practical alternative was proposed. The cavity angular velocity was replaced by the angular velocity of the wetted vortex at a radius equal to the cavity radius. Although this approach is physically incorrect, it does give an accurate description of the tip vortex cavity resonance frequency in this case. It could be applied to any loading distribution, by using the appropriate circulation and roll-up parameter $\beta$.

\section{Discussion}

A $90^{\circ}$ sector of the flow field was taken as a representative for the description of the tip vortex. Due to the obstructions in the image, no accurate comparison could be made to the contour average based on the full field of view. Care should be taken when comparing the findings from the present study to other results.

The vector quality in the part of the sector that is closest to the center was affected by the presence of the cavity interface. Variations in cavity diameter caused some of the interrogation areas to be inside the cavity. The resulting poor correlation contribution is expected to be less significant because of the use of correlation averaging. The interrogation areas outside the cavity will result in a higher correlation peak and contribute more to the final vector. In any case the interrogation areas outside the bounds of the cavity diameter variation in Figs. 16 and 17 should not be affected.
Several speculations exist on the nature of the flow field surrounding a vortex cavity. Bosschers (2015) has derived an analytical formulation for the azimuthal velocity distribution around a two-dimensional viscous cavitating vortex. The result is referred to as a cavitating Lamb-Oseen vortex. This was derived using the appropriate jump relations for the stresses at the cavity interface. The shear stress at the vaporliquid interface is approximately zero. The resulting zero shear stress condition creates a small region of solid body rotation, as was found in the experimental results of Figs. 16 and 17.

Alternatively, Gaussian vortex formulations were proposed by Choi and Ceccio (2007) and Choi et al. (2009). These differed from the previous formulation with an additional parameter describing the azimuthal velocity at the cavity interface. A range of interfacial azimuthal velocities from 0 to the velocity of a wetted vortex was possible.

The physical behavior of the model by Bosschers (2015) is very similar to the present measurements. However, the wetted Lamb-Oseen vortex, which made an analytical treatment of the physics possible, poorly describes the wetted vortex. It is therefore also incapable of quantitatively describing the velocity field around the vortex cavity. A detailed model of the flow field around vortex cavitation should be based on more realistic vortex models. Unfortunately a physical analytic treatment is then probably no longer possible.

The same comments apply to the potential flow vortex, that is the basis of the tip vortex cavity resonance frequency model (Pennings et al. 2015). The origin of the resonance frequency could not have been analytically explained without a potential flow basis. The quantitative usefulness of the resonance frequency model, including the corrected wetted flow input, should further be evaluated in practice on real propeller flows.

\section{Conclusion}

A method, based on the vortex center identification and correlation averaging, was successfully applied to stereo particle image velocimetry around a wing tip vortex in the presence and absence of cavitation. This procedure provided results with sufficient resolution and accuracy for use in the following detailed observations.

The Proctor vortex model, which is restricted to the region outside the viscous core, is a good general description of a wing tip vortex in close streamwise proximity of the tip. It only relies on the empirical $\beta$ parameter that is dependent on the wing loading distribution and streamwise distance. It can also describe the cavity size as a function of the cavitation number.

A general trend is found in the effect of cavitation on the tip vortex flow field. The cavity interface is surrounded by a region of retarded azimuthal velocity. The velocity gradient close to the interface approximates solid body rotation. 
Bosschers (2015) analytically derived the region of solid body rotation from a zero shear stress condition at the cavity interface. The region of retarded flow decreases in size for larger cavities until the flow field is equal to that of a wetted vortex.

The tip vortex cavity resonance frequencies found by Pennings et al. (2015) could be described, by using the angular velocities of a wetted vortex at the cavity radius as a model input for the cavity angular velocity. This approximation of the physics shows the limits of the potential flow modeling approach.

Acknowledgments The experimental work has been funded by Lloyd's Register Foundation, as part of a cooperation in the International Institute for Cavitation Research. The suggestions of Jerke Eisma and Sedat Tokgöz, at the Laboratory of Aero \& Hydrodynamics, related to the sum of correlation reprocessing, have been monumental in the improvement of the experimental data quality. The proof reading efforts of Arati Gurung were greatly appreciated.

Open Access This article is distributed under the terms of the Creative Commons Attribution 4.0 International License (http://creativecommons.org/licenses/by/4.0/), which permits unrestricted use, distribution, and reproduction in any medium, provided you give appropriate credit to the original author(s) and the source, provide a link to the Creative Commons license, and indicate if changes were made.

\section{References}

Abbott I, Doenhoff A (1959) Theory of wing sections. Dover Publications, New York

Arndt R, Keller A (1992) Water quality effects on cavitation inception in a trailing vortex. J Fluids Eng 114:430-438

Arndt R, Arakeri V, Higuchi H (1991) Some observations of tip-vortex cavitation. J Fluid Mech 229:269-289

Astolfi J, Fruman D, Billard J (1999) A model for tip vortex roll-up in the near field region of three-dimensional foils and the prediction of cavitation onset. Eur J Mech B/Fluids 18(4):757-775

Batchelor G (1964) Axial flow in trailing line vortices. J Fluid Mech 20:645-658

Bhagwat M, Ramasamy M (2012) Effect of tip vortex aperiodicity on measurement uncertainty. Exp Fluids 53:1191-1202

Bosschers J (2015) An analytical solution for the viscous flow around a 2-D cavitating vortex. J Fluids Eng (submitted)

Boulon O, Callenaere M, Franc J, Michel J (1999) An experimental insight into the effect of confinement on tip vortex cavitation of an elliptical hydrofoil. J Fluid Mech 390:1-23

Burnham D, Hallock J (1982) Chicago monostatic acoustic vortex sensing system. Technical report DOT-TSC-FAA-79-18, IV, U.S. Department of Transportation

Chang N, Ganesh H, Yakushiji R, Ceccio S (2011) Tip vortex cavitation suppression by active mass injection. J Fluids Eng 133(11):111301. doi:10.1115/1.4005138

Choi J, Ceccio S (2007) Dynamics and noise emission of vortex cavitation bubbles. J Fluid Mech 575:1-26

Choi J, Hsiao C, Chahine G, Ceccio S (2009) Growth, oscillation and collapse of vortex cavitation bubbles. J Fluid Mech 624:255-279

del Pino C, Parras L, Felli M, Fernandez-Feria R (2011) Structure of trailing vortices: comparison between particle image velocimetry measurements and theoretical models. Phys Fluids 23:013602. doi:10.1063/1.3537791
Devenport W, Rife M, Liapis S, Follin G (1996) The structure and development of a wing-tip vortex. J Fluid Mech 312:67-106

Dreyer M, Decaix J, Münch-Alligné C, Farhat M (2014) Mind the gap: a new insight into the tip leakage vortex using stereo-PIV. Exp Fluids 55:1849. doi:10.1007/s00348-014-1849-7

Felli M, Falchi M (2011) Propeller tip and hub vortex dynamics in the interaction with a rudder. Exp Fluids 51:1385-1402

Felli M, Roberto C, Guj G (2009) Experimental analysis of the flow field around a propeller-rudder configuration. Exp Fluids 46:147-164

Felli M, Camussi R, Di Felice F (2011) Mechanisms of evolution of the propeller wake in the transition and far fields. J Fluid Mech 682:5-53

Foeth E (2008) The structure of three-dimensional sheet cavitation. Ph.D. thesis, Delft University of Technology, Delft, The Netherlands

Fruman D, Cerrutti P, Pichon T, Dupont P (1995) Effect of hydrofoil planform on tip vortex roll-up and cavitation. J Fluids Eng 117:162-169

Gerz T, Bryant FHW, Köpp F, Frech M, Tafferner A, Winckelmans G (2005) Research towards a wake-vortex advisory system for optimal aircraft spacing. Comptes Rendus Physique 6:501-523

Graftieaux L, Michard M, Grosjean N (2001) Combining PIV, POD and vortex identification algorithms for the study of unsteady turbulent swirling flows. Meas Sci Technol 12:1422-1429

Higuchi H, Quadrelli J, Farell C (1987) Vortex roll-up from an elliptic wing at moderately low Reynolds numbers. AIAA J 25(12):1537-1542

Lee T (2011) PIV study of near-field tip vortex behind perforated Gurney flaps. Exp Fluids 50:351-361

Maines B, Arndt R (1997) The case of the singing vortex. J Fluids Eng 119:271-276

Mei R (1996) Velocity fidelity of flow tracer particles. Exp Fluids 22:1-13

Meinhart C, Wereley S, Santiago J (2000) A PIV algorithm for estimating time-averaged velocity fields. J Fluids Eng 122:285-289

Pennings P, Bosschers J, Westerweel J, van Terwisga T (2015) Dynamics of isolated vortex cavitation. J Fluid Mech 778:288-313

Prasad A, Adrian R, Landreth C, Offutt P (1992) Effect of resolution on the speed and accuracy of particle image velocimetry interrogation. Exp Fluids 13:105-116

Proctor F (1998) The NASA-Langley wake vortex modelling effort in support of an operational aircraft spacing system. In: Proceedings of the 36th Aerospace Sciences Meeting \& Exhibit, AIAA, Reno, NV, USA

Scarano F, van Wijk C, Veldhuis L (2002) Traversing field of view and AR-PIV for mid-field wake vortex investigation in a towing tank. Exp Fluids 33:950-961

Stanislas M, Okamoto K, Kähler C (2003) Main results of the First International PIV Challenge. Measurement. Sci Technol 14:R63-R89

Westerweel J (2008) On velocity gradients in PIV interrogation. Exp Fluids 44:831-842

Wijngaarden E, Bosschers J, Kuiper G (2005) Aspects of the cavitating propeller tip vortex as a source of inboard noise and vibration. In: Proceedings of the ASME fluids engineering division summer meeting and exhibition, Houston, TX, USA

Wu J, Ma H, Zhou M (2006) Vorticity and vortex dynamics. Springer, New York

Zhang H, Zhou Y, Whitelaw J (2006) Near-field wing-tip vortices and exponential vortex solution. J Aircr 43(2):445-449

Zverkhovskyi O (2014) Ship drag reduction by air cavities. Ph.D. thesis, Delft University of Technology, Delft, The Netherlands 